Revue scientifique sur la conception et l'aménagement de l'espace

\title{
Quand les franges urbaines se mettent au vert: la nature aux marges de la métropole lyonnaise
}

When Urban Fringes Go Green: Nature on the Edges of the City of Lyons

Inès Méliani

\section{OpenEdition}

\section{Journals}

Édition électronique

URL : http://journals.openedition.org/paysage/10313

DOI : $10.4000 /$ paysage. 10313

ISSN : 1969-6124

\section{Éditeur :}

École nationale supérieure du paysage de Versailles-Marseille, Institut national des sciences appliquées Centre Val de Loire - École de la nature et du paysage, École nationale supérieure d'architecture et de paysage de Bordeaux, École nationale supérieure d'architecture et de paysage de Lille, Agrocampus Angers

\section{Référence électronique}

Inès Méliani, «Quand les franges urbaines se mettent au vert : la nature aux marges de la métropole lyonnaise », Projets de paysage [En ligne], 13 | 2015, mis en ligne le 31 décembre 2015, consulté le 04 septembre 2020. URL : http://journals.openedition.org/paysage/10313 ; DOI : https://doi.org/ $10.4000 /$ paysage. 10313

Ce document a été généré automatiquement le 4 septembre 2020

Projets de paysage 


\section{Quand les franges urbaines se mettent au vert : la nature aux marges de la métropole lyonnaise}

When Urban Fringes Go Green: Nature on the Edges of the City of Lyons

Inès Méliani

1 Aujourd'hui lieux de vie de près d'un quart de la population française, les espaces périurbains se sont transformés depuis l'après-guerre et au long de plusieurs décades, se développant de façon spontanée et le plus souvent anarchique pour constituer des territoires spécifiques, les franges urbaines, dont les caractéristiques modifient profondément la traditionnelle opposition rural-urbain.

2 Longtemps, ces "franges urbaines" n'ont été regardées que comme des extensions désordonnées, résultat de l'étalement du tissu urbain sous la poussée d'une pression démographique excessive liée à l'afflux des ruraux vers la ville, que le périmètre urbain ne suffit plus à contenir. À la périphérie des villes on observe alors un phénomène d'urbanisation de la campagne: aux espaces naturels et agricoles qui jusqu'ici constituaient la trame des zones périurbaines se mêlent désormais des éléments structurels et infrastructurels spécifiques des modes de vie citadins, dans une dynamique qui contribue à modeler des territoires d'un type nouveau se caractérisant par une grande diversité paysagère, économique et sociale. Dans sa politique d'aménagement, la métropole lyonnaise a très tôt pris en compte ce phénomène, cherchant, dans un premier temps, à organiser le désordre. C'est ainsi que, dès les années 1970, les schémas d'urbanisme - essentiellement le schéma d'aménagement de la métropole Lyon-Saint-Étienne-Grenoble (1971) - répondent à la volonté de maîtriser la croissance par une planification globale intégrant ces nouveaux territoires.

Puis à compter des années 1980, l'évolution des modes de vie et des mentalités des citadins impulse, sous l'effet de la forte densification du tissu urbain, un mouvement contraire : au pouvoir d'attraction de la ville se substitue l'aspiration à s'éloigner de la ville pour se rapprocher de la nature. Le regard sur les franges urbaines s'inverse alors : ces zones envisagées jusqu'ici comme génératrices de contraintes que l'on peine à 
organiser acquièrent leurs lettres de noblesse. Conjugué, à partir des années 1990, à l'émergence des préoccupations écologistes dans le champ politique, ce phénomène change la donne. Ainsi en 1992, l'élaboration du schéma directeur de l'agglomération lyonnaise (SDAL) traduit une vision positive des franges urbaines, où l'espace naturel devient un élément stratégique d'attractivité et de qualité de vie du territoire.

4 Aujourd'hui, les franges urbaines constituent des territoires dont les aménageurs se préoccupent pour répondre aux problématiques des villes contemporaines et concevoir la ville de demain. Devenir une métropole-nature en préservant son agriculture et ses espaces naturels et en les replaçant au centre du projet urbain constitue un défi, d'autant plus actuel qu'il correspond à des préoccupations montantes de la société, telles que le bien-être des habitants ou l'écologie. Les politiques urbaines commencent à intégrer sensiblement cette idée, et la réglementation de ces dernières années vient confirmer l'amorce de ces changements. Au cœur de ce projet essentiel pour la ville, une question récurrente se pose : comment valoriser l'interface rural-urbain pour qu'il devienne la référence systématique, armature durable mais dynamique et évolutive de la métropole?

5 Concernant la question des franges urbaines, l'agglomération lyonnaise fait figure de pionnière par une politique d'aménagement originale à cet égard : très tôt en effet les documents d'urbanisme ont pris en compte ces territoires dans leur spécificité, traduisant le souci d'orienter leur destination, leurs fonctionnalités, leur inscription territoriale. Il est intéressant alors d'examiner quelles sont, dans leur développement chronologique, les stratégies mises en œuvre dans l'agglomération lyonnaise pour préserver puis contrôler la lisibilité des franges urbaines. Et tout d'abord, comment se sont développées les franges urbaines? Quels sont les secteurs de l'agglomération lyonnaise où l'institutionnalisation de la nature a fixé les franges urbaines? Quelles formes paysagères se sont alors développées sur ces contacts? Quels outils sont mobilisés?

6 Cet article a ainsi pour ambition d'éclairer les nouvelles dynamiques qui se jouent au sein des franges urbaines de l'agglomération lyonnaise en examinant les ressorts historiques, économiques et de stratégie politique à l'œuvre.

\section{Les franges urbaines dans les documents d'urbanisme : no man's land non maîtrisables ou potentiel pour l'avenir?}

$7 \mathrm{Au}$ sein de l'agglomération lyonnaise, les franges urbaines constituent des espaces ambivalents dont la physionomie résulte d'une extension spontanée du tissu urbain, sous la poussée de deux flux contradictoires. Il y a dans un premier temps l'exode rural, qui vient grossir la ville de telle façon que ses limites reculent du centre vers la périphérie: la ville s'étend au-delà de son périmètre formel pour répondre à des besoins en habitat, en équipements et en infrastructures. Il y a ensuite le mouvement des citadins vers la campagne : les franges urbaines ont alors pour rôle de répondre aux besoins de nature, assurant ainsi la présence de la nature en ville. Elles sont aussi témoin de l'histoire et du patrimoine de la ville, et assurent les entrées et sorties de ville en direction des espaces périurbains et au-delà. 
D'une façon générale, les franges urbaines constituent des espaces très composites qui - pour paraphraser François Ost, Jean Rémy et Luc Van Campenhoudt (1993) - ne correspondent ni à des champs, ni à des forêts, ni à des parcs, ni à des espaces verts. Il en est ainsi des franges urbaines de l'agglomération lyonnaise, où les espaces seminaturels se conjuguent aux espaces naturels et agricoles. La première des caractéristiques permettant de les définir a trait à leur statut tout à la fois marginal et éminemment patrimonial. Le site du Petit Fontanière dans le quartier de La Mulatière à Lyon, qui allie espaces de nature et éléments du patrimoine historique et culturel de la ville, en constitue un bon exemple. Ce sont des espaces-lisières qui, situés aux confins de la ville, créent du lien avec les espaces urbains et agricoles. Pour les nommer, François Ost, Jean Rémy et Luc Van Campenhoudt (1993) utilisent les termes évocateurs d'«[...] espaces résiduels, espaces par défaut, des contre-espaces, des espaces de transition ".

9 À Lyon, la préoccupation liée à la présence des espaces d'entre-deux ne date pas d'hier. Elle est contemporaine de l'accélération du phénomène de densification des villes. Au cours de la seconde moitié du $\mathrm{xx}^{\mathrm{e}}$ siècle et sous la pression d'une forte croissance démographique principalement liée à l'exode rural, l'urgence alors a été de faire de la maîtrise de l'étalement urbain une priorité, avec, pour corollaire, le souci de réfléchir à la place et à l'enjeu du traitement des espaces naturels situés en périphérie de ville. Le desserrement urbain a conduit à une urbanisation diffuse dans les secteurs de frange : quartiers pavillonnaires, habitat dispersé, petits collectifs se sont développés sur ces espaces intermédiaires. Les politiques se préoccupent alors dès les années 1960 de la question des limites de la ville. Au sein de l'agglomération lyonnaise en effet, depuis le début de cette période, la planification urbaine s'est principalement focalisée sur l'espace urbain, ne considérant les espaces de nature situés en limite de ville qu'en tant que facteur de contrôle de l'expansion urbaine. Si bien que dans les documents de planification urbaine - schéma de l'organisation d'étude et d'aménagement de l'aire métropolitaine Lyon-Saint-Étienne-Grenoble (Oream, 1971), puis dans le schéma directeur d'aménagement et d'urbanisme (Sdau, 1978) -, les espaces semi-naturels ne sont entendus que pour contenir la ville, l'espace rural étant considéré quant à lui comme une réserve d'extension urbaine.

Figure 1. La nature aux marges de la métropole lyonnaise dans les documents de planification urbaine

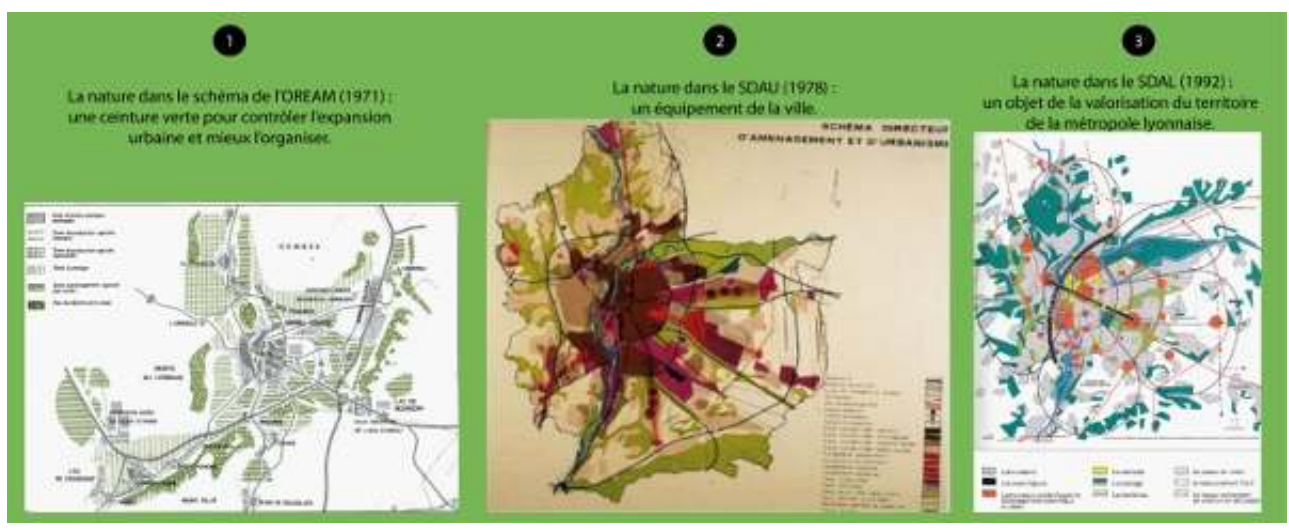

Un changement de direction progressif depuis la ceinture verte (1- autour de la ville), puis la nature équipement (2 - autour et dans la ville) et jusqu'au déploiement (3 - depuis la ville-centre vers l'Est lyonnais), 2013. 
10 urbain a conduit à une prise de conscience et, afin d'infléchir la tendance et de maitriser l'extension urbaine, deux axes ont été privilégiés. Le premier vise à réorganiser le foncier sur la base du critère de proximité pour les usagers, en renforçant les fonctions de centralité dans les centres urbains. Le second axe vise à préserver une structure verte, correspondant aux territoires ruraux et aux espaces non urbanisés - les espaces naturels et agricoles périurbains, soit environ $40 \%$ de la surface de l'agglomération lyonnaise, dénommée également ceinture ou coupure verte. Ils constituent de véritables infrastructures naturelles, des outils de régulation de la forme et de l'expansion urbaine et des éléments essentiels de l'attractivité économique et résidentielle de l'agglomération.

11 Le SDAL de 1992 traduit une inversion du regard sur les espaces verts et les questions environnementales. Les espaces naturels situés en périphérie de ville y sont pris en compte en tant qu'éléments de qualité de vie, la proximité de la nature étant alors perçue comme une chance et non plus comme une contrainte. Durant ces quarante années, tout en continuant à s'inscrire dans une logique de projet, la planification intègre le phénomène d'expansion de la ville, ce qui conduit à institutionnaliser la nature, et donc à fixer les franges urbaines. Et de fait, c'est l'institutionnalisation de cette nature qui va fixer les franges urbaines de la ville. C'est ainsi par exemple qu'apparaît la question de la protection d'un certain nombre de sites: le massif des Monts-d'Or (le Nord de Lyon), le Franc Lyonnais (le Nord de Lyon), les îles de MiribelJonage (le Nord-Est), les Balmes viennoises (le Sud-Est) et une partie des vallons de l'Ouest. Ils sont classés en sites dits « inaltérables» (Amzert et Cottet-Dumoulin, 2000). De même, d'autres sont identifiés en tant qu'espaces de loisirs pour les habitants de la métropole. Ainsi pensée, la planification urbaine tend alors à intégrer la demande sociale d'espaces récréatifs dans les programmes d'aménagement urbain. Il en résulte qu'aujourd'hui, les franges urbaines présentent une grande diversité de paysages et de pratiques habitantes.

12 Parallèlement au schéma directeur, deux révisions (1985 et 1988) du plan d'occupation des sols (POS) du Grand Lyon s'imposent en réponse à l'expansion urbaine de Lyon. En 1992 débute une troisième révision du POS, qui repose sur deux orientations principales mais fondamentales pour formaliser et pérenniser les limites de l'agglomération lyonnaise et leur statut dans le paysage.

13 Il est d'abord question de préserver le caractère naturel des grands sites paysagers, tels les balmes (pentes des collines), les collines de la Croix-Rousse ( $4^{\mathrm{e}}$ arrondissement), la colline de Fourvière ( $5^{\mathrm{e}}$ arrondissement), celles de la Duchère et de Saint-Rambert ( $9^{\mathrm{e}}$ arrondissement) ainsi que le plateau du $5^{\mathrm{e}}$ arrondissement. C'est à la suite de cette troisième révision du POS qu'une démarche pour inclure ces espaces, publics ou privés, dans la catégorie des espaces à protéger est lancée. Les extensions des bâtiments existants seront strictement délimitées. Pour préserver les points de vue depuis la ville vers les collines ou depuis les collines vers la ville, la hauteur des constructions sur les quais est réduite, et la constructibilité sur les crêtes réglementée. Les périmètres de protection et de mise en valeur des espaces naturels et agricoles périurbains (Penap) prendront corps de cette volonté de protéger ces grands sites paysagers. 
Figure 2. Balmes le long du quai Fulchiron à Lyon (quais de Saône)

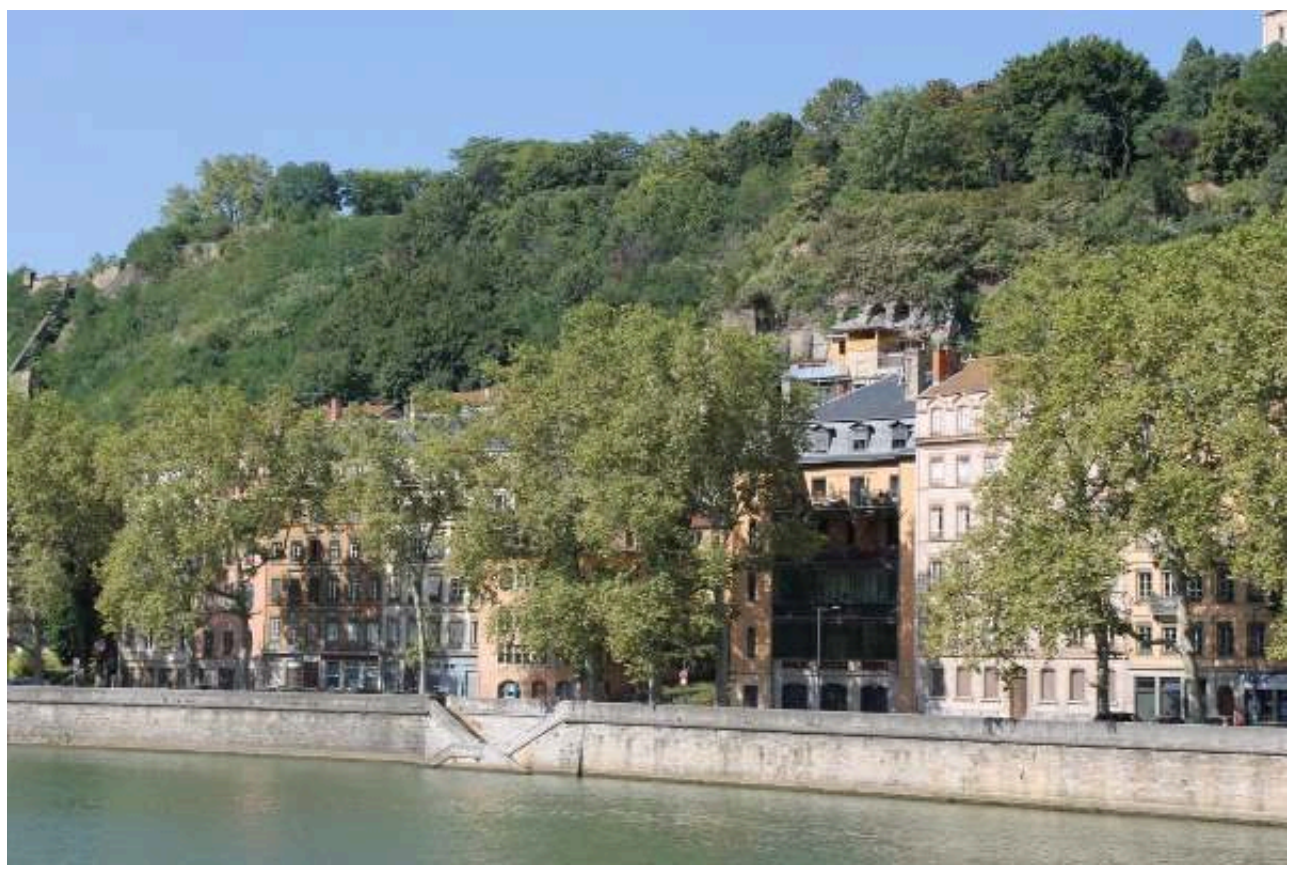

Source : Inès Méliani, 2015.

14 Il est ensuite question de protéger et de développer les espaces boisés dans et autour de la ville. C'est à ce titre que le POS envisage la création de grands parcs à dominante arborée et de parcs dits intermédiaires. De manière sous-jacente, le concept de trame verte émerge : le prolongement paysager depuis le parc de la Tête d'Or vers le Rhône et l'aménagement du parc de Gerland sont mis à l'étude, de même qu'un itinéraire piétonnier qui, prolongeant le parc des Hauteurs de Fourvière situé en surplomb des rives de la Saône, relie différents sites d'intérêt végétal, culturel ou visuel, en coupant à travers espaces verts publics et parcelles privées.

\section{Les franges urbaines : atout de valorisation du territoire? L'« inversion du regard »}

Les années 1990 sont donc marquées par un changement radical dans la façon dont les aménageurs considèrent les franges urbaines de l'agglomération lyonnaise, dont ils ont désormais une vision valorisante - ce que les urbanistes expriment en parlant d'«inversion du regard». En effet, jusque-là considérées comme des espaces de transition où l'urbanisation diffuse fragmentait de façon anarchique le pourtour de l'agglomération, les franges urbaines apparaissent désormais comme des territoires importants au regard de l'organisation spatiale de la ville: dès lors, aux yeux des politiques, s'efforcer d'articuler l'espace urbanisé et l'espace vert dans les franges urbaines apparaît à l'évidence comme le meilleur moyen et la véritable solution pour éviter le mitage urbain. C'est d'ailleurs ce principe que défendra, quelques années plus tard, Pierre Donadieu (1998). Selon lui, les espaces périurbains ne doivent pas seulement être considérés comme lieux de flânerie pour les citadins. Il convient plutôt de les intégrer dans une réflexion sur « les relations entre la ville et la campagne, entre la culture urbaine et le monde agricole ". Et à l'heure où aménageurs et urbanistes se 
préoccupent de la destination des espaces agricoles périurbains en termes de « ceinture verte ", il pose la question : «[...] Au lieu de chercher en vain à contrôler la croissance de la ville par des réseaux de ceintures, de fronts et d'espaces verts, pourquoi ne pas construire le tissu urbain avec les espaces agricoles et forestiers? Pourquoi l'agriculture périurbaine ne pourrait-elle pas être considérée par les aménageurs comme un outil d'urbanisme capable d'organiser durablement le territoire des cités ?»

C'est dans le contexte de ce changement de vision sur les franges urbaines qu'intervient la création des « projets nature » : ceux-ci sont élaborés avec le passage d'une politique de protection foncière à la nécessité de mettre en œuvre des outils de gestion et d'animation des espaces naturels et agricoles.

En 1992, la communauté urbaine de Lyon élabore les « projets nature » dans le cadre de la charte de l'écologie urbaine. Les projets nature sont présentés par le Grand Lyon comme « un outil de gestion durable et de mise en valeur des espaces de la trame verte, un instrument financier, un espace de concertation » et sont remarquables par la " diversité importante dans le contenu de leurs actions, leur dynamisme, leur ancienneté, la taille et la nature des territoires concernés, les partenaires engagés ». Ils voient le jour au travers de la charte de l'écologie urbaine qui, parue en 1992, devient le document de référence concrétisant la politique menée par le Grand Lyon en matière d'environnement.

Au début des années 1990, onze projets nature sont alors conçus, concernant quatre secteurs : la région des Monts-d'Or (avec les vallons de Serres et des Planches, le vallon des Échets et les monts d'Or); les coteaux de l'Ouest lyonnais (avec le vallon de l'Yzeron, le plateau de Méginand et ses abords - c'est-à-dire les vallons du Ribes - et le plateau des Hautes-Barolles); les grandes plaines de l'Est lyonnais (avec les Grandes Terres et la partie nord du V-vert) ; le long des axes fluviaux (avec Miribel-Jonage, les îles et lônes du Rhône - secteur aval de Lyon).

Dans le cadre de ces différents projets, le Grand Lyon a réalisé de nombreuses actions de sensibilisation et de préservation des zones naturelles et agricoles. Élaborés avec différents partenaires - qui peuvent être la commune, le département ou une autre collectivité (tel un syndicat intercommunal), et pour la plupart coordonnés avec le conseil général - ces projets présentent des réalisations très diverses. Parmi les actions de préservation, on peut mentionner les inventaires de la faune et de la flore, la plantation de haies, la création de sentiers nature, de mares. Quant à la démarche de sensibilisation, elle est principalement axée sur l'éducation à l'environnement, chaque « projet nature » créant son propre programme en relation avec les associations locales socioculturelles ou de protection de la nature, avec pour objectif la découverte du patrimoine naturel local (faune, flore, paysages, agriculture...)

Par sa portée politique, économique et sociale d'abord, par ses rôles spatiaux (reliant les territoires, dépassant le carcan de leurs échelles conceptuelles) et historiques ensuite, le "projet nature " ressemblerait alors, à y regarder de près, à un modèle de développement durable pour les métropoles européennes. 
Figure 3. Des « projets nature » et des sentiers de promenade pour les loisirs verts dans les franges urbaines du Grand Lyon

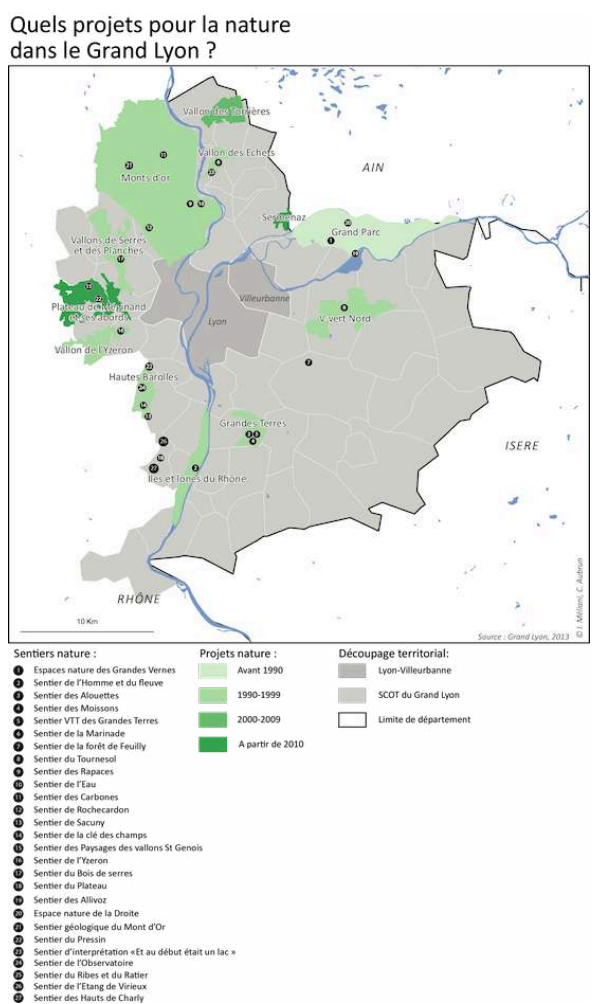

Le nom de ces « sentiers nature » présente de l'intérêt pour une lecture territoriale et paysagère de la métropole lyonnaise. La diversité des objets végétaux (Grandes Vernes), animaux (alouettes, rapaces), minéraux (sentiers géologiques, sentier du Plateau), fluviaux (sentier de I'Yzeron, sentier de l'Eau, de l'Homme et du fleuve) ou correspondant à des localisations (rues des Moissons, de la Droite), traduit la richesse des préoccupations et des représentations des différentes catégories et le lien avec le territoire, amorçant les préoccupations de patrimonialisation de la nature aux marges de la ville.

21 Cette politique d'aménagement qui consiste à articuler l'espace urbanisé et l'espace vert situé dans les franges urbaines se retrouve logiquement dans le schéma de cohérence territoriale (Scot) de l'agglomération lyonnaise.

L'État prévoit que chaque territoire se dote d'un Scot d'ici le $1^{\mathrm{er}}$ janvier 2017. Le Scot se trouve au croisement des échelles régionale et locale. Il fournit de grandes orientations et vise à coordonner les politiques menées en matière de développement économique, d'urbanisme, d'habitat, d'implantations commerciales et de déplacements. Toutefois, il ne fournit pas de carte de destination des sols. À Lyon, c'est le Syndicat mixte d'études et de programmation de l'agglomération lyonnaise (Sepal) qui élabore le Scot en concertation avec les communes concernées. Il fonctionne grâce à un appel de fonds auprès de ses adhérents, qui acquittent une somme fixée au prorata de leur population. Les options adoptées pour le Scot de l'agglomération lyonnaise s'articulent autour de cinq principaux thèmes de réflexion, parmi lesquels la préservation des espaces naturels et agricoles s'inscrit comme une question majeure. Son document de présentation prône «l'inversion du regard ». Selon cette logique, les espaces naturels, agricoles et forestiers y sont considérés comme des espaces "pleins» et non plus comme des espaces «vides », car, selon le Scot de l'agglomération lyonnaise, «[...] la traditionnelle opposition entre la ville et la nature n'a plus lieu d'être. Les espaces naturels, agricoles et forestiers forment désormais un tout avec les espaces urbanisés ». Ce principe d'emboîtement d'échelle permet alors de rapprocher la ville et la 
campagne, suivant la logique selon laquelle les espaces naturels, agricoles et forestiers des franges urbaines sont désormais reconnus comme une infrastructure structurante du territoire, comme il a été souligné plus haut. Tout comme celui de Montpellier, le Scot de l'agglomération lyonnaise semble vouloir considérer les espaces naturels ou cultivés comme des éléments essentiels de valorisation du territoire. De fait, en articulant compositions urbaines et continuités écologiques, il érige les trames vertes et bleues en éléments structurants du projet urbain, afin de mieux ordonnancer la ville (Ascher, 1995). Et, au-delà de la trame verte et bleue, il intègre des outils - liaisons, coupures, corridors verts - qui viennent s'entre-tisser dans le système de trame, lui conférant ainsi tout sa cohérence.

Même si, à Lyon, les questions relatives aux espaces agricoles périurbains sont prises en compte de façon systémique dès les années 1970 - on parlait alors de "grenier de la ville » et de " coupure verte »-, ainsi qu'au début des années 1990, sous la mandature de Michel Noir - une " mission Écologie » est créée, fixant déjà les rôles de la ceinture verte -, ce n'est qu'avec le Grenelle de l'Environnement (2007) qu'apparaît la notion de trame verte, et que les espaces naturels et agricoles périurbains sont envisagés sous l'angle de leur multifonctionnalité : s'ils sont espaces de production, ils sont aussi zones d'accueil pour un public de citadins en mal d'évasion dans la nature.

Selon l'article L371-16 du code de l'environnement, la trame verte comprend « [...] tout ou partie des espaces protégés [...] ainsi que les espaces naturels importants pour la préservation de la biodiversité, les corridors écologiques constitués des espaces naturels ou semi-naturels, ainsi que des formations végétales linéaires ou ponctuelles conçues pour diminuer la fragmentation et la vulnérabilité des habitats naturels et habitats d'espèces, et prendre en compte leur déplacement dans le contexte du changement climatique.» La trame verte comprend les espaces définis à l'article L211-14 du code de l'environnement, à savoir la couverture végétale située « le long de certains cours d'eau, sections de cours d'eau et plans d'eau de plus de dix hectares » et que « l'exploitant ou, à défaut, l'occupant ou le propriétaire de la parcelle riveraine est tenu de mettre en place et de maintenir ».

L'objectif de la trame verte et bleue est à la fois de contribuer à la conservation des habitats naturels afin de "[...] permettre aux espèces animales et végétales dont la préservation ou la remise en bon état constitue un enjeu national ou régional de se déplacer pour assurer leur cycle de vie et favoriser leur capacité d'adaptation » et de participer «au bon état écologique des masses d'eau ». Le décret du 27 décembre 2012 précise enfin que la trame verte et bleue est à considérer en tant «qu'outil d'aménagement durable du territoire ».

$\mathrm{Au}$ sein de l'agglomération lyonnaise, une cartographie de la trame verte est réalisée afin d'en suivre l'évolution. Sa prise en compte dans l'élaboration du plan local d'urbanisme (PLU) vise à en garantir la préservation à l'horizon 2030. Ainsi, 8700 hectares sont classés en zone $\mathrm{N}$ - naturelle - et 11500 hectares sont en zone A agricole. Les liaisons vertes et bleues sont pensées afin de structurer le territoire du nord de l'agglomération vers le sud, mais également du sud vers le nord. Le Rhône et la Saône constituent l'axe territorial du réaménagement de la trame verte et bleue, de sorte que, du parc de Miribel-Jonage situé au nord au parc de Gerland au sud, en passant par la Feyssine, le parc de la Tête d'Or et les berges du Rhône, se dessinent les pièces essentielles des nouvelles structures spatiales de la ville. 
Figure 4. Le système de trame verte structure le territoire

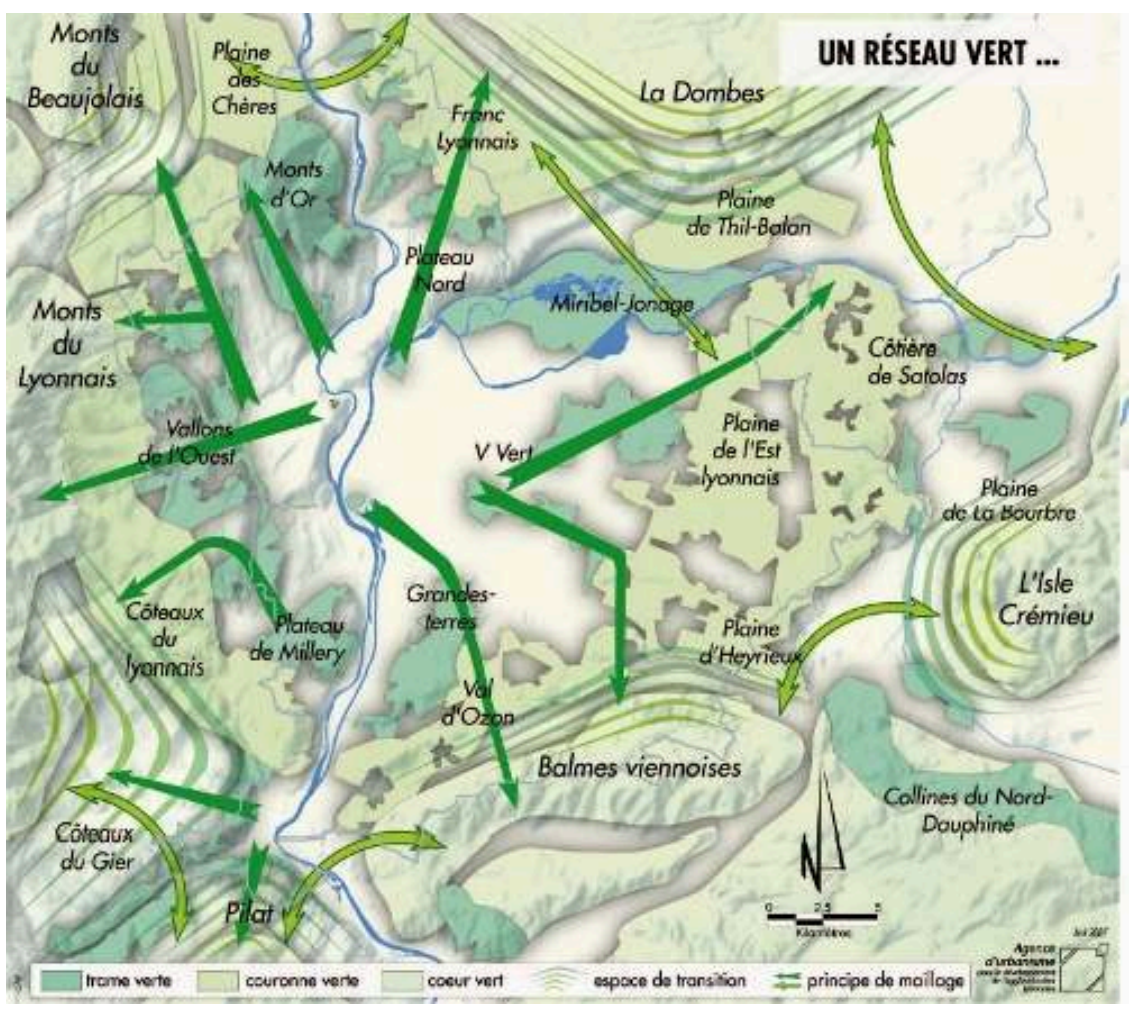

La carte permet de jouer sur le linéaire, le ponctuel et le zonal. Les zones de transition de l'agglomération lyonnaise et l'articulation des liaisons vertes en territoire urbain et en territoire naturel et agricole y sont particulièrement visibles.

Source : Agence d'urbanisme de Lyon, 2008.

27 C'est ainsi qu'au cœur de la trame verte et bleue de la métropole lyonnaise coexistent différentes générations de parcs urbains et périurbains : assurant le lien entre le «dedans » et le "dehors", ils constituent des espaces de loisirs tout en contenant le développement des franges urbaines dans une certaine limite.

À cet égard, le parc de Miribel-Jonage constitue un exemple parmi les plus significatifs. En effet, de l'état initial du site à sa physionomie actuelle, l'histoire de son développement rend bien compte des destins successifs assignés aux franges urbaines en fonction des modifications de perception dont elles ont pu être l'objet, et dont résultent à la fois les différents usages et les formes paysagères qui s'y sont développées.

Créé en 1968 et d'une superficie de 200 hectares - dont 350 hectares de plans d'eau et 700 hectares de milieux protégés dans sa partie nord-est -, le parc de Miribel-Jonage est aujourd'hui considéré comme le plus grand parc périurbain de France et l'un des plus grands d'Europe. Il accueille chaque année près de 3,5 millions de visiteurs. Historiquement, l'espace de son site actuel était, depuis le Moyen Âge et jusqu'au début $\mathrm{du} \mathrm{xx}^{\mathrm{e}}$ siècle, constitué de terres agricoles. Vaste zone humide, le développement d'autres usages y était peu envisageable. C'est à la faveur de la création du réservoir du Grand Large dans le cadre de la canalisation du Rhône qu'ont pu se développer des usages de loisirs. À l'origine perçu comme une «zone sauvage " permettant « l'accès à une forme de liberté à quelques minutes de la ville » (Amzert et Cottet-Dumoulin, 2000), le parc de Miribel-Jonage est à la fois périurbain, intercommunal et 
interdépartemental. De 1959 à 1985, de nombreux aménagements du site sont réalisés : activités nautiques sur un lac de 1500 hectares et création d'équipements sportifs, dont un rocher d'escalade en 1977, des courts de tennis en 1978, un centre d'accueil enfance en 1980, une piste cyclable en 1982, un parcours de golf en 1985. Un centre sportif payant, Planète tonique, propose plus de 20 activités sur une quinzaine d'hectares. Classé «zone naturelle inaltérable » en 1992 au SDAL, le parc de Miribel-Jonage est devenu un parc nature en 1993. Aujourd'hui, espace récréatif aux dimensions impressionnantes et qui s'inscrit dans les pratiques de loisirs des habitants de l'agglomération lyonnaise, le grand parc de Miribel-Jonage permet aussi, par son enracinement sur l'espace périurbain, de prévenir la progression des franges urbaines vers le nord des zones urbaines, et ainsi donc d'en contenir les limites. Sa gestion s'articule en outre autour d'un enjeu essentiel : la cohabitation entre la base de loisirs et les autres usages, avec notamment huit zones naturelles d'intérêt écologique, faunistique et floristique (Znieff). La création de sentiers de découverte vient renforcer sa vocation pédagogique et, faisant écho à la réputation de Lyon comme ville culturelle, l'art et la nature s'y combinent à travers deux disciplines: les arts de la danse et du spectacle et les arts plastiques.

\section{Conclusion}

Les franges urbaines, qui forment ces nouvelles urbanités (Luginbühl, 2007), résultent des effets importants de la métropolisation et des grands projets d'aménagement. L'élaboration d'une ceinture verte au sein de l'agglomération lyonnaise illustre les nouveaux défis concernant ces espaces intermédiaires, ni tout à fait urbains, ni tout à fait ruraux. Aujourd'hui à Lyon, les franges urbaines s'inscrivent plus franchement dans l'organisation du territoire, avec une volonté affirmée de mettre en cohérence et de manière plus systématique les espaces de proximité immédiate. Au travers du réaménagement des fronts d'eau et autres coulées vertes, la linéarité urbaine s'est imposée comme figure nouvelle : en témoignent les tracés des corridors écologiques, des berges du Rhône, de la Saône, de la confluence Rhône-Saône, etc. Penser ces nouvelles formes de ville bouscule quelque peu l'échelle des valeurs entre le construit et le non-construit.

31 Au sens strict, l'urbanisme communal pourrait encore améliorer sa façon de considérer et de prendre en compte les franges urbaines. Encore faut-il avoir le courage de prendre le parti d'assigner à certains lieux une vocation d'inconstructibilité, de manière à rendre possibles des continuités écologiques. Or, aujourd'hui encore, laisser un terrain indemne de toute construction est trop souvent perçu comme une anomalie se traduisant par un manque à gagner. Et bien souvent encore, c'est la spéculation foncière et le court terme qui déterminent les priorités d'action.

Mais il reste indéniable que, au-delà des polémiques et des stratégies financières ou politiques, le fait de fixer les franges urbaines favorise le sentiment de confort des habitants (loisirs, promenades), contribue à contenir l'étalement urbain, et préserve le caractère écologique de la ville. La frange urbaine en dessine l'avenir. 


\section{BIBLIOGRAPHIE}

Ascher, F., Métapolis ou l'avenir des villes, Paris, Odile Jacob, 1995.

Autran, S., Les infrastructures vertes à l'épreuve des plans d'urbanisme. L'agglomération lyonnaise, la construction d'une stratégie, Lyon, Certu, 2004.

Amzert, M., Cottet-Dumoulin, L., « Du "sauvage" à "l'inaltérable” : les conditions sociales de création d'un espace naturel en milieu urbain. Le cas du parc de Miribel-Jonage ", Géocarrefour, vol. 75, nº 4, p. 283-292, 2000, URL : http://www.persee.fr/doc/

geoca_1627-4873_2000_num_75_4_2480.

Donadieu, P., Campagnes urbaines, Arles, Actes Sud, Coll. « Nature », 1998, 219 p.

Luginbühl, Y., Nouvelles Urbanités, Nouvelles Ruralités en Europe, Bruxelles, PIE Peter Lang, 2007.

Luginbühl, Y., « Perception paysagère des espaces en déprise et des boisements spontanés des terres agricoles », Ingénieries - EAT, p. 25-29, 1999.

Méliani, I., « La nature en ville, entre protection, communication et patrimonialisation : approches géographiques dans les territoires du Grand Lyon ", thèse de doctorat de géographie sous la direction de Paul Arnould, ENS de Lyon - UMR 5600 CNRS-Environnement Ville Société, 2013, 296 p.

Ost, F., La Nature hors la loi, Paris, La Découverte, 2003.

Ost, F., Rémy, J., Van Campenhoudt, L., Entre ville et nature, les sites semi-naturels, Bruxelles, Publications des facultés universitaires Saint-Louis, 1993.

Toublanc, M., Bonin, S., «Planifier les trames vertes dans les aires urbaines : une alliance à trouver entre paysagisme et écologie ", Développement durable et territoires, vol. 3, $\mathrm{n}^{\circ} 2$, mis en ligne en juillet 2012, URL : https://developpementdurable.revues.org/9347.

\section{RÉSUMÉS}

La physionomie de la métropole lyonnaise se caractérise par un fort étalement du tissu urbain qui se traduit, notamment depuis l'après-guerre, par une constante artificialisation des sols et la disparition de milliers d'hectares de surfaces naturelles. La ville-centre a peu à peu absorbé les communes alentour, laissant subsister par endroits des îlots d'espaces naturels et agricoles. Longtemps considérés comme terrains résiduels potentiellement constructibles, ceux-ci constituent aujourd'hui une catégorie d'espaces s'inscrivant, en tant que valeur ajoutée, dans les projets publics d'aménagement. Très tôt pris en compte dans la planification urbaine, ces espaces en mutation, où formes paysagères, pratiques habitantes et gouvernances se sont développées de multiples façons, ont contribué à fixer les franges urbaines. Les documents d'urbanisme de l'agglomération lyonnaise témoignent de ce phénomène d'institutionnalisation d'une nature récréative, à travers maints exemples qui viennent éclairer la problématique des franges urbaines: ainsi le réaménagement en trame verte du linéaire des berges $d u$ Rhône ou l'élaboration des "projets nature " et, plus significatifs encore, les aménagements du parc de Miribel-Jonage au nord, ou du parc de Gerland au sud de Lyon, sur une ancienne friche industrielle. Cette étude montre comment les espaces de transition entre Lyon et campagne, longtemps vus comme complexités mal maîtrisables, ont acquis le statut d'atout pour la mise en 
œuvre d'une politique d'aménagement visant la qualité de vie et répondant aux nouveaux enjeux écologiques.

The physiognomy of the Lyon metropolis is characterised by intense urban sprawl which has led to the disappearance of thousands of hectares of natural land. The city centre has gradually absorbed the outlying municipalities leaving behind islands of natural and agricultural lands. For a long time, these remaining spaces were seen as potential building land but today they are considered as spaces providing added value to public development projects. Taken into account upstream in the urban planning process these changing spaces, which have given rise to a variety of different landscapes, modes of use and methods of governance, have contributed to defining the urban fringes of Lyons. Urban planning documents of the Lyons agglomeration are evidence of the institutionalisation of a form of recreational nature through many examples which shed light on the problems relating to urban fringes: the redevelopment of the network of green spaces on the banks of the River Rhone, the deployment of "Nature Projects", and the two major projects of the Miribel-Jonage Park to the north and the Gerland Park in a former brownfield to the south of the city. This study shows how the transitional spaces between Lyons and the countryside, long considered as complex areas to manage, are now perceived as assets in an urban planning policy aimed at improving quality of life and addressing new environmental issues.

\section{INDEX}

Keywords : Lyons metropolis, nature, urban fringe, urban development plan, reverse perspective, urban planning documents

Mots-clés : métropole lyonnaise, nature, frange urbaine, schéma d'aménagement urbain, inversion du regard, documents d'urbanisme

\section{AUTEUR}

\section{INÈS MÉLIANI}

Inès Méliani. Docteure en géographie, spécialiste des questions environnementales en milieu urbain, elle est chercheure associée au laboratoire Environnement Ville Société, UMR5600 CNRS et rattachée à l'équipe biogéophile de l'École normale supérieure de Lyon. Ses principaux travaux s'intéressent à la place et au statut de la nature en milieu urbain en lien avec les questions de marketing territorial. La métropole lyonnaise constitue son terrain d'étude privilégié. ines.meliani[at]ens-lyon[dot]fr 